\title{
La Actividad Innovadora por Género en América Latina: un estudio de patentes
}

\author{
Rosa María Morales Valera \\ Facultad de Ciencias Económicas y Sociales de la Universidad de Carabobo, Venezuela
}

Domingo Alberto Sifontes Fernandez

Facultad de Ciencias Económicas y Sociales de la Universidad de Carabobo, Venezuela

Recebido: 03/02/2012 Versão Revisada (entregue): 30/10/2012 Aprovado: 22/11/2012

\begin{abstract}
RESUMEN
La Red Iberoamericana de Ciencia, Tecnología e Innovación muestra que en la mayoría de los países de América Latina existe relativamente poca información acerca de las actividades de ciencia y tecnología por género. Esta investigación intenta aportar en el tema de género y tecnología a través del estudio de la participación por género en la actividad patentadora de América Latina. Se hace un análisis comparativo de las patentes por género desde 1990 hasta el 2006. Se analizan 2082 patentes registradas por Argentina, Brasil, México, Colombia, Cuba, Perú, Chile y Venezuela en la Oficina de Patentes de los Estados Unidos (USPTO). Se encontró que el $20 \%$ de los inventos involucra participación del género femenino. Los países con mayor desigualdad de género en la producción de patentes son Perú, Argentina y México. Las mujeres participan más en las áreas de Química y Metalurgia.
\end{abstract}

\footnotetext{
Este artículo es producto del proyecto "Ciencia, Tecnología e Innovación en América Latina" financiado por el Consejo de Desarrollo Científico y Humanístico de la Universidad de Carabobo (CDCH-UC) Subvención N²009-001. Los autores agradecen a los asistentes al Seminario ALTEC 2011 y a dos árbitros anónimos por comentarios. Nuestros agradecimientos se hacen extensivos a Angélica Rodríguez y Héctor Monasterios por asistencia de investigación y comentarios.
} 
Rosa María Morales Valera, Domingo Alberto Sifontes Fernandez

PALABRAS Claves | Innovación; Ciencia; Género; América Latina; Investigación Tecnológica

Códigos JEL | J16; O31; O54

Innovative Activity by Gender: a Latin American patent study

\begin{abstract}
The Red Iberoamericana de Ciencia, Tecnología e Innovación shows that there is relatively few information about science and technology activities by gender in most Latin American countries. This research aims to contribute on the issue of gender and technology through the study of gender participation in patenting activity in Latin America. Patents from 1990 to 2006 are analyzed. The sampled countries are Argentina, Brazil, Mexico, Colombia, Cuba, Peru, Chile and Venezuela. The source of information is the United States Patent and Trademarks Office (USPTO). This study finds that $20 \%$ of the inventions involve female participation. Countries with greater gender inequality in innovative activities are Peru, Argentina and Mexico. Women are more involved in the areas of Chemistry and Metallurgy.
\end{abstract}

KEYWORDS | Innovation; Science; Gender; Latin America; Technological Research

JEL-CODES | J16; O31; O54

\title{
1. Introducción
}

Los estudios de género dentro de la actividad científica en América latina cobran cada vez mayor importancia cuando se intenta explicar la desigualdad entre hombres y mujeres en el desarrollo de la ciencia en el continente. Esfuerzos importantes se realizan desde la "Cátedra Regional UNESCO Mujer, Ciencia y Tecnología en América latina" y la Red Iberoamericana de Ciencia y Tecnología en las cuales estudios previos (ESTÉBANEZ, 2002; VESSURI; CANINO, 2006; VESSURI; CANINO;RAUSELL, 2004; LÁSCARIS, 2004) destacan la importancia de darle un enfoque de género a la construcción de indicadores de ciencia y tecnología. 
Las estadísticas de la Red Iberoamericana de Ciencia y Tecnología Iberoamericana e Interamericana (RICYT) para América Latina muestran que en la mayoría de los países latinoamericanos, la participación de investigadoras excede el tercio del total de investigadores por país entre 1990 y el 2006, llegando incluso a superar el 50\% en países como Argentina, Cuba y Venezuela para algunos años. El informe: "El estado de la Ciencia” publicado por RICYT en su versión del año 2009 corrobora que la participación de la mujer en la actividad científica del continente ha venido en aumento en los últimos 10 años, en efecto, para el año 2007, el 48\% de los investigadores en América latina eran mujeres. Sin embargo, la base de datos de la Red de Información de Política Científica de la UNESCO muestra que el empleo femenino por industria no excede el tercio del total de trabajadores empleados en el sector industrial y existe una tendencia a la baja para la mayoría de los países de América Latina en los últimos años. Indicando que si bien es cierto que la participación de la mujer en la actividad científica ha aumentado, esto no se observa de la misma forma en el resto de las actividades profesionales.

Los indicadores para el área científica y tecnológica en América latina relacionados con el género se enfocan en la participación de la actividad científica, más no en su aporte a la actividad de producción industrial. Los datos por género son escasos en el caso de la actividad innovadora orientada hacia la comercialización, es decir, la actividad patentadora. En este sentido, el presente estudio pretende contribuir en esta dirección, al estudiar la productividad por género de la actividad innovadora en nueve países de América latina: Argentina, Brasil, Chile, Colombia, Costa Rica, Cuba, México, Perú y Venezuela.

El estudio comienza con una breve revisión de estudios previos en el área de género y patentes para diferentes industrias y países. Luego aborda aspectos metodológicos que permitieron clasificar por género los datos disponibles. Se presenta una sección de resultados en términos de participación, contribución e impacto de las patentes por género de los inventores de acuerdo a países, organizaciones y áreas de especialización en la actividad innovadora. Finalmente, se discuten los resultados.

\section{Revisión de la literatura}

En años recientes la literatura sobre género y patentes ha ido creciendo. Existen distintos enfoques para tratar el tema. Una corriente de la literatura se enfoca hacia el estudio de cómo se crea conocimiento y su influencia en la diferencias en la participación por género y otra hacia el estudio de la desigualdad sin cuestionar 
la forma en que se crea conocimiento (HALBERT, 2006). En cualquier caso, sin discriminar el enfoque que se use para estudiar género, ciencia y tecnología, cada vez se muestra más interés por el tema de la desigualdad de género en la actividad patentadora debido a que los resultados muestran una brecha bastante amplia a favor de los hombres. Así, Naldi, Luzi, Valente y Vaninni (2004) estudian el desempeño científico tecnológico por género para 6 países de Europa, tomando en cuenta las patentes y publicaciones realizadas. Generando una base de datos que las distribuyó por género, país, sector industrial y disciplina de estudio. Los resultados muestran que la participación, contribución y el número de mujeres es menor en patentes que en publicaciones. Las industrias de mayor contribución de las mujeres patentando son farmacia y química y el área donde menor cantidad de publicaciones presentan es matemática. También para Europa, Kugele (2007) y Busolt y Kugele (2009) estudian la participación y productividad investigadora de las mujeres ampliando la muestra de Naldi et al. (2004). Los resultados arrojan que Lituania es el país con mayor porcentaje de mujeres dedicadas a la invención (23\%) y Austria el de menor (5\%). Existe un porcentaje menor de mujeres inventoras respecto a las mujeres investigadoras y Alemania que es el país con mayor actividad inventiva de la región y la participación de mujeres es de $6 \%$, ubicándose por debajo de la media Europea.

Por su parte, Whittington y Smith-Doerr, (2005) estudian las disparidades de los resultados de los esfuerzos innovadores tanto en el sector industrial y académico para el área de ciencias de la vida. Encuentran que las mujeres producen menos que los hombres en ambos sectores y que esta diferencia es constante en el tiempo. A pesar que las mujeres producen menos patentes, la calidad y el impacto de estas son iguales o superiores a la producida por los hombres. La desigualdad de género varía de acuerdo al sector donde los inventores trabajen: el sector industrial en general, el sector industrial de ciencias de la vida solamente o el mundo académico, siendo menor para el sector industrial de ciencias de la vida que en los otros dos sectores, no existiendo diferencias significativas en las tasas a las cuales tanto hombres como mujeres patentan a través de los dos sectores. Respecto a la forma organizacional, Whittington y Smith-Doerr, (2008) encuentran que en estructuras más flexibles y con más tendencias a la colaboración como aquellas existentes en el sector de ciencias de la vida (biotecnología, química), las mujeres tienen más posibilidades de patentar que en organizaciones jerárquicas y más rígidas existentes en los otros sectores. Sin embargo, "el canal de transmisión" por el cual la forma organizacional impacta a la desigualdad de género en la producción de patentes no es concluyente. 
Por su parte Ding, Murray, y Stuart, (2006) también para el área de ciencias de la vida, determinan las causas de las diferencias de género en la actividad patentadora solo para el sector académico, encontrando que las mujeres patentan un $40 \%$ de lo que los hombres lo hacen y que la desigualdad ha disminuido a lo largo del tiempo pero sigue siendo alta.

Para la industria biotecnológica norteamericana, McMillan (2009) integra tres bases de datos existentes que le permitieron clasificar 1903 patentes por género de acuerdo a si la patente era producida solo por mujeres, solo por hombres o intervenían ambos. Los resultados muestran que el $4 \%$ de las patentes fueron descubiertas solo por mujeres y que de un total de 5000 inventores, menos de 900 eran mujeres, sin embargo, en las patentes en las que intervienen hombres y mujeres, el total de inventoras es del 36\%.Con respecto al número promedio de inventores por patentes encontró que es menor cuando participan sólo mujeres y es mayor cuando participan ambos. A pesar de las mujeres patentar menos, la calidad de sus patentes, medida por la cantidad de citas recibidas, es mayor a la de los hombres.

Por su parte, Frietsch, Haller, Funken-Vrohlings, y Grupp (2009) estudian los "patrones de patentamiento" y publicaciones por género para 14 países de tres continentes. Concluyen que la participación femenina en los últimos 10 años ha aumentado en ambos ámbitos: patentes y publicaciones, sin embargo, existen diferencias sustanciales entre cada uno de los países que posiblemente se asocien con la estructura tanto del sector industrial como del sector académico en cada uno de los países del estudio y el impacto que esto tiene en el mercado laboral.

Mauleón y Bordons (2010) calculan indicadores de actividad tecnológica por género en España para comparar la participación y contribución de los inventores durante el período 1990-2005, analizando la participación por género en las patentes con solicitantes españoles. Concluyen que existe un incremento en las solicitudes de patentes durante el período de estudio, los hombres patentes más que las mujeres, sin embargo, se muestra un incremento en la participación femenina y las mujeres tienen mayor actividad en el sector público en las áreas de Necesidades Humanas y Química y Metalurgia. Asimismo, la participación de hombres y mujeres en una misma patente se incrementó.

\section{Metodología}

La unidad de análisis de este estudio es la patente. La fuente de información es la Oficina de Patentes de los Estados Unidos (USPTO). Se eligió la base de datos de 
la USPTO porque esa base de datos es la que contiene muchas de las innovaciones registradas en el mundo. Esto es debido a que si un inventor quiere evitar que otro patente antes, debe intentar registrar su invento en aquel país donde la ocurrencia de dicho suceso sea mayor, y ese país es Estados Unidos (MANI, 2001). Además de la cantidad de patentes registradas, la USPTO posee una base de datos donde se detalla el documento de la patente de manera minuciosa, ofreciendo mayor información por documento que otra base de datos. Finalmente, los inventores latinoamericanos tienen una mayor propensión a patentar en Estados Unidos que en Europa por la cercanía geográfica.

La muestra de países en estudio incluye a Argentina, Brasil, Chile, Colombia, Costa Rica, Cuba, México, Perú y Venezuela. Se estudian 2082 patentes otorgadas a estos países durante el período 1990-2006. Se investigan las patentes otorgadas porque estas son conocimiento del cual los inventores ya pueden apropiarse de sus beneficios. En algunos análisis del estudio el número de observaciones difiere porque no se puede determinar el género del inventor o bien porque no se pudo determinar la clasificación internacional por sección o tipo de entidad dueña de la patente.

El documento de la patente de la base de datos de la USPTO provee información sobre distintos elementos, en este estudio se consideraron los siguientes:

- número de la patente;

- fecha cuando se otorga la patente;

- nombre del inventor;

- nombre del individuo o entidad dueña de la patente;

- nombre del país que alberga al individuo o entidad dueña de la patente;

- clasificación internacional por sección;

- referencias hechas por otros inventores de la patente en estudio.

El número de la patente se usa para identificar la unidad de observación de manera única y así evitar duplicar los documentos. La fecha de otorgamiento de la patente se utiliza para clasificar los documentos de acuerdo al año. El nombre del inventor es clave para este estudio porque a partir de este se deduce el género del inventor. La forma a través de la cual se dedujo el género fue a partir del primer nombre, en caso de que no se pudiese deducir el género a partir del primer nombre, se revisaba el segundo nombre o se buscaba en internet, si eran nombres en idiomas distintos al español.

El nombre del individuo o entidad dueña de la patente es usado para indagar acerca del esquema de propiedad de la entidad. Si es una universidad nacional, un 
laboratorio, un instituto o una empresa del Estado, entonces se codifica la patente como de propiedad pública, por el contrario si es una compañía o un individuo entonces se establece que la patente es de propiedad privada, finalmente si el dueño de la patente es una empresa mixta, la propiedad es mixta.

El nombre del país que alberga al individuo o entidad dueña de la patente es la categoría ACN (asignee country) de la USPTO y sirvió para categorizar a cual país se asignó la patente. El derecho de propiedad intelectual sobre una invención puede ser de una o varias entidades, cuando es de una sola entidad que se localiza en un determinado país, se contabiliza esa patente a ese país, pero cuando es de varias entidades que pertenecen a distintos países pues se comparte la asignación. Estos casos son la excepción y no llegan a representar el 2\% de la muestra.

La clasificación internacional por sección (Sección CIP) es una herramienta que usa la Organización Mundial de Propiedad Intelectual para catalogar a las patentes de acuerdo a sectores. Mauleon y Bordons (2010) explican que la clasificación internacional permite categorizar a las patentes de acuerdo a la tecnología que emplean. Las siguientes son las categorías que sirven para clasificar las patentes de forma amplia:

- necesidades humanas: esta categoría contiene los inventos que corresponden a las necesidades corrientes de la vida, puede incluir inventos en el sector alimentos, higiene y salud;

- operaciones y transporte: esta categoría incluye inventos en el sector transporte, impresión, operaciones de mezclado y separación de elementos, nanotecnología;

- química y metalurgia: en esta sección se incluyen inventos en el área de química orgánica e inorgánica: incluidas industrias del petróleo, gas y azúcar. Inventos en el área de bioquímica, microbiología, enzimología, ingeniería genética y metalurgía también son incluidos en esta sección;

- textiles y papel: los inventos incluidos en esta sección son los relativos a la industria de los textiles y papelera;

- construcciones fijas: se refiere a los inventos relativos a edificaciones, perforaciones y minería;

- ingeniería mecánica: inventos expresados a través de maquinaría, bombas, ingeniería en general, iluminación y calefacción se incluyen en esta sección;

- física: se clasifican en esta sección a los inventos asociados a los instrumentos, bien sean de medición, chequeo o computacionales. En esta sección también se encuentran los inventos en ingeniería nuclear; 
- electricidad: en esta categoría se incluyen a los aparatos de generación, conversión y distribución eléctrica, así como circuitos electrónicos y comunicaciones eléctricas.

Ahora bien, las referencias hechas por otros inventores de la patente en estudio se utilizan para medir el impacto de esa patente. El número de referencias hechas por otros constituye las citas que tiene una patente en particular, esto permite indicar cuán importante es la patente para la comunidad científica y tecnológica.

Los indicadores que se usan en este estudio son:

- participación por género: este indicador refleja en cuantas patentes (tanto en forma absoluta como de forma relativa) participan solo hombres, solo mujeres o ambos sexos. La participación por género se calcula por país, por secciones y por esquema de propiedad de la patente;

- contribución por género femenino: este indicador muestra en cuanto contribuyen las mujeres en la producción de una patente. Este índice incluye las patentes en las que participan solo mujeres o aquellas en que participan ambos géneros. Siguiendo a Frietsch et al. (2009) se calculó asumiendo una distribución uniforme, es decir que el aporte es igual en ambos sexos. Si en una patente hay 7 inventores y 3 de ellos son mujeres, entonces la contribución del género femenino es de 3/7. Se calcula por país y para toda la muestra;

- impacto promedio de las patentes por género: este indicador mide en promedio cuanto se citan las patentes por participación de género. Se hace un conteo de citas por cada patente, posteriormente se clasifican las patentes de acuerdo al género y luego se calcula el promedio de citas por género. Así, cuando se hace referencia al impacto promedio de las patentes de solo mujeres, se está dividiendo el total de citas de todas las patentes donde hay solo inventoras entre el total de patentes de esas inventoras. Este indicador se estima para cada país en estudio y para toda la muestra. El conteo de citas se hace hasta el 2008 para disminuir sesgos con las patentes más jóvenes.

\section{Resultados}

La Tabla 1 clasifica el porcentaje de patentes registradas por país durante el período de estudio. Se observa que Brasil es el país que más patentes registradas en la USPTO presenta con el $47 \%$ y Perú el que menor aporte presenta con $1 \%$. 
TABLA 1

Porcentaje de patentes registradas

Países seleccionados - 1990-2006

\begin{tabular}{l|c}
\hline \multicolumn{1}{c|}{ Países } & $\%$ \\
\hline Argentina & 7,0 \\
Brasil & 47,0 \\
Chile & 3,0 \\
Colombia & 2,0 \\
Costa Rica & 2,0 \\
Cuba & 2,0 \\
México & 23,0 \\
Perú & 1,0 \\
Venezuela & 13,0 \\
Total & 100,0 \\
\hline
\end{tabular}

Fuente: Oficina de Patentes de los Estados Unidos (USPTO). Elaboración de los autores.

La Tabla 2 muestra que del total de patentes analizadas para el período de estudio en el 3,62\% participan sólo mujeres, en el 79,44\% solo hombres y en el $16,94 \%$ la participación involucra al menos a un hombre o una mujer. Durante este período las patentes registradas logran ser mayores en 2006 que en 1990, sin embargo, los registros de 2006 no representan el mayor número de patentes por género, esto se evidencia para el año 2003. El año con menor producción de inventos que involucren solo mujeres se registró en 1991, 1994 y 1995 con 1 patente, para solo hombres y mixta fue en 1990 con 54 y 4 respectivamente. Se observa también como las patentes registradas sólo por hombres son mayores a aquellas en las cuales participa una mujer y como las de participación mixta superan a las que solo involucran mujeres. Sin embargo, para el año 2003, se observa una mayor participación de solo mujeres en el registro de inventos, reafirmando la propensión al alza de registros en el período 2002-2004 (si se realiza una serie móvil centrada trianual se puede observar de forma más precisa esa tendencia). 
Rosa María Morales Valera, Domingo Alberto Sifontes Fernandez

TABLA 2

Participación anual de acuerdo a género en las patentes registradas en la USPTO Países seleccionados (1) - 1990-2006

\begin{tabular}{r|c|c|c|c}
\hline Año & Solo mujeres & Solo hombres & Mixta & Total \\
\hline 1990 & 3 & 54 & 4 & 61 \\
1991 & 1 & 65 & 6 & 72 \\
1992 & 4 & 56 & 9 & 69 \\
1993 & 3 & 74 & 17 & 94 \\
1994 & 1 & 61 & 9 & 71 \\
1995 & 1 & 92 & 13 & 106 \\
1996 & 4 & 87 & 10 & 101 \\
1997 & 5 & 90 & 15 & 110 \\
1998 & 8 & 84 & 27 & 119 \\
1999 & 5 & 126 & 25 & 156 \\
2000 & 7 & 134 & 30 & 171 \\
2001 & 2 & 108 & 26 & 136 \\
2002 & 7 & 111 & 34 & 152 \\
2003 & 11 & 145 & 43 & 199 \\
2004 & 5 & 144 & 27 & 176 \\
2005 & 3 & 84 & 19 & 106 \\
2006 & 4 & 108 & 32 & 144 \\
Total & $\mathbf{7 4}$ & $\mathbf{1 6 2 3}$ & $\mathbf{3 4 6}$ & $\mathbf{2 0 4 3}$ \\
\% & $\mathbf{4 , 0}$ & $\mathbf{7 9 , 0}$ & $\mathbf{1 7 , 0}$ & $\mathbf{1 0 0 , 0}$ \\
\hline
\end{tabular}

Fuente: Oficina de Patentes de los Estados Unidos (USPTO). Elaboración de los autores.

(1) Argentina, Brasil, Chile, Colombia, Costa Rica, Cuba, México, Perú, Venezuela.

La Tabla 3 clasifica la participación por género y país de las patentes registradas en la USPTO durante el período de estudio. Se observa que Venezuela es el país donde las mujeres presentan una mayor participación con el 45,2\% de sus patentes: $6,9 \%$ realizada solo por mujeres y $38,3 \%$ con participación mixta, por su parte, Cuba presenta el mayor porcentaje de participación femenina, 83\%, a pesar de no presentar patentes realizadas solo por mujeres. Aparte de Perú, Cuba es otro país donde los hombres participan en el 100\% del total de sus patentes registradas.

Del total de patentes en las que existe participación de mujeres (solo mujeres y mixtas) la participación mixta es mayor que la de solo mujeres. Para la participación de hombres (solo hombres y mixtas) la participación de solo hombres es mayor a la mixta. 
Respecto al total de patentes por género, se observa que Brasil es el país que mayor cantidad de patentes registradas posee en cada categoría: solo mujeres, solo hombres y mixta. Perú y Cuba no presentan patentes registradas solo por mujeres, Cuba es el país con menos registros realizados solo por hombres y Perú nuevamente no presenta patentes de participación mixta, debido a que todas sus patentes son registradas solo por hombres.

TABLA 3

Participación por género de las patentes registradas Países seleccionados - 1990-2006

\begin{tabular}{|c|c|c|c|c|c|c|c|c|}
\hline \multirow[t]{2}{*}{ Países } & \multicolumn{2}{|c|}{$\begin{array}{l}\text { Patentes solo } \\
\text { por mujeres }\end{array}$} & \multicolumn{2}{|c|}{$\begin{array}{l}\text { Patentes solo } \\
\text { por hombres }\end{array}$} & \multicolumn{2}{|c|}{$\begin{array}{c}\text { Patentes con } \\
\text { participación } \\
\text { mixta }\end{array}$} & \multicolumn{2}{|c|}{ Total } \\
\hline & N. abs. & $\%$ & N. abs. & $\%$ & N. abs. & $\%$ & N. abs. & $\%$ \\
\hline Argentina & 4 & 2,61 & 137 & 89,54 & 12 & 7,84 & 153 & 100,00 \\
\hline Brasil & 33 & 3,47 & 791 & 83,09 & 128 & 13,45 & 952 & 100,00 \\
\hline Chile & 3 & 4,48 & 51 & 76,12 & 13 & 19,40 & 67 & 100,00 \\
\hline Colombia & 1 & 3,13 & 26 & 81,25 & 5 & 15,63 & 32 & 100,00 \\
\hline Costa Rica & 2 & 4,55 & 37 & 84,09 & 5 & 11,36 & 44 & 100,00 \\
\hline Cuba & 0 & 0,00 & 8 & 17,02 & 39 & 82,98 & 47 & 100,00 \\
\hline México & 12 & 2,60 & 411 & 88,96 & 39 & 8,44 & 462 & 100,00 \\
\hline Perú & 0 & 0,00 & 12 & 100,00 & 0 & 0,00 & 12 & 100,00 \\
\hline Venezuela & 19 & 6,93 & 150 & 54,74 & 105 & 38,32 & 274 & 100,00 \\
\hline Total & 74 & 3,62 & 1.623 & 79,44 & 346 & 16,94 & 2.043 & 100,00 \\
\hline
\end{tabular}

Fuente: Oficina de Patentes de los Estados Unidos (USPTO). Elaboración de los autores.

En el Gráfico 1 se observa que por secciones CIP, el área en la que más patentan los hombres solos es Operaciones y Transporte, seguido por Química y Metalurgia e Ingeniería Mecánica, mientras que las mujeres solas patentan más en el área de Química. La participación mixta es más común también en el área de Química. Se observa una marcada diferencia entre las patentes elaboradas solo por hombres y aquellas en las que participan solo mujeres para todas las secciones CIP, llegando a existir hasta una diferencia de 334 patentes para el área de Operaciones y Transporte. Por otra parte, la diferencia entre el número de patentes otorgadas sobre inventos por secciones CIP donde participan solo hombres y las patentes donde hay participación de ambos géneros no es tan marcada como en el caso anterior. 
La mayor diferencia por secciones CIP, se tiene también en el área de Operaciones y Transporte pero no llega a 300.

\section{GRÁFICO 1}

Patentes asignadas, por género, según secciones CIP Países seleccionados (1) - 1990-2006
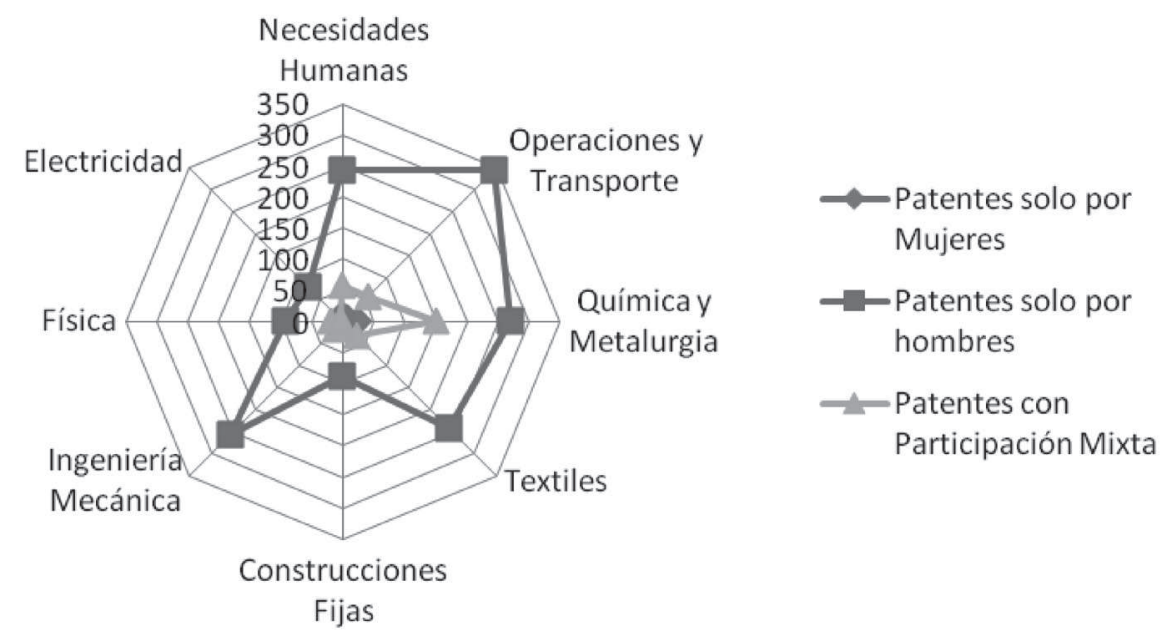

Fuente: Oficina de Patentes de los Estados Unidos (USPTO). Elaboración de los autores. (1) Argentina, Brasil, Chile, Colombia, Costa Rica, Cuba, México, Perú, Venezuela.

Con respecto al esquema de propiedad de las organizaciones que patentan, la Tabla 4 muestra que el $77,46 \%$ de las patentes se realizan en organizaciones privadas, el $19,97 \%$ en organizaciones públicas y el 2,57\% en organizaciones con esquema de propiedad mixta. La participación es mayoritariamente de solo hombres para los tres esquemas de propiedad: $86,10 \%$ privado, $74,51 \%$ mixto y $52,64 \%$ público. Se observa entonces una mayor desigualdad de género en las organizaciones privadas que en las públicas. Esta desigualdad se podría explicar usando el "Efecto Ciencias de la Vida" expuesto por Whittington y Smith-Doerr (2008). Las empresas del sector privado que patentan en la mayoría de los países de América Latina no están orientadas al sector de ciencias de la vida (biotecnología, química) mientras que las del sector público si se focalizan en ese sector. ${ }^{1}$ Por lo tanto, las empresas

1 Un ejemplo del patentamiento del sector químico, es la industria petrolera en Venezuela que opera a través de una empresa pública que posee el mayor registro de invenciones del país y es donde se observa una mayor actividad patentadora del género femenino. 
públicas de algunos países de América Latina al estar orientadas al sector químico tienden a tener ambientes con mayor colaboración y más flexibilidad facilitando la disminución de la brecha entre géneros.

TABLA 4

Participación por género de inventores de patentes, según el esquema de propiedad de la institución a la cual se le asigna la patente Países seleccionados (1) - 1990-2006

\begin{tabular}{l|c|c|c}
\hline $\begin{array}{c}\text { Esquema de } \\
\text { propiedad }\end{array}$ & $\begin{array}{c}\text { Patentes solo } \\
\text { por mujeres }\end{array}$ & $\begin{array}{c}\text { Patentes solo } \\
\text { por hombres }\end{array}$ & $\begin{array}{c}\text { Patentes con } \\
\text { participación mixta }\end{array}$ \\
\hline Público & 24 & 209 & 164 \\
Privado & 49 & 1326 & 165 \\
Mixto & 1 & 38 & 12 \\
\hline
\end{tabular}

Fuente: Oficina de Patentes de los Estados Unidos (USPTO). Elaboración de los autores.

(1) Argentina, Brasil, Chile, Colombia, Costa Rica, Cuba, México, Perú, Venezuela.

La contribución por género femenino en la producción de patentes mide cuanto aportan las mujeres en la producción de una patente e incluye las patentes con participación solamente de mujeres o con participación mixta. Se observa en la Tabla 5 que los países donde las mujeres contribuyen más en el registro de una patente son Cuba, Venezuela y Chile.

TABLA 5

Contribución por género femenino en registro de patentes Países seleccionados - 1990-2006

\begin{tabular}{|c|c|}
\hline Países & $\begin{array}{c}\text { Contribución por } \\
\text { mujeres (\%) }\end{array}$ \\
\hline Argentina & 5,49 \\
\hline Brasil & 9,64 \\
\hline Chile & 12,08 \\
\hline Colombia & 6,70 \\
\hline Costa Rica & 7,24 \\
\hline Cuba & 39,45 \\
\hline México & 5,83 \\
\hline Perú & 0,00 \\
\hline Venezuela & 21,06 \\
\hline
\end{tabular}

Fuente: Oficina de Patentes de los Estados Unidos (USPTO). Elaboración de los autores. 
Es de observar que a pesar de que Cuba no tiene patentes solo con participación de mujeres, es el país donde la mujeres contribuyen más, esto es debido a la contribución de las mujeres en la producción de patentes donde participan ambos géneros. Los países donde existe menos contribución de las mujeres son Perú y México. Perú es un país donde las mujeres no participan en el registro de patentes en la USPTO, eso explica por qué no contribuyen. Es de destacar que en ningún país de la muestra la contribución promedio alcanza el $40 \%$ y solo en Cuba alcanza el 33\%.

En la Tabla 6 se observa que el área de Química y Metalurgia es en la que los inventores sin distingo de género patentan más si se toma en cuenta todos los países de la muestra. Chile, Cuba, México y Venezuela son los países que coinciden con esta característica, mientras que en Colombia, Costa Rica y Argentina los inventores registran más patentes en el área de Necesidades Humanas. Brasil no presenta esta característica ya que tiene más patentes en Operaciones y Transporte.

TABLA 6

Número de patentes por secciones CIP Países seleccionados - 1990-2006

\begin{tabular}{|c|c|c|c|c|c|c|c|c|c|}
\hline Países & $\begin{array}{l}\text { Necesi- } \\
\text { dades } \\
\text { Humanas }\end{array}$ & $\begin{array}{c}\text { Opera- } \\
\text { ciones y } \\
\text { Transporte }\end{array}$ & $\begin{array}{l}\text { Química } \\
\text { y Meta- } \\
\text { lurgia }\end{array}$ & $\begin{array}{l}\text { Tex- } \\
\text { tiles }\end{array}$ & $\begin{array}{c}\text { Const. } \\
\text { Fijas }\end{array}$ & $\begin{array}{c}\text { Inge- } \\
\text { niería } \\
\text { Mecánica }\end{array}$ & Física & $\begin{array}{l}\text { Electri- } \\
\text { cidad }\end{array}$ & Total \\
\hline Argentina & 45 & 36 & 26 & 16 & 5 & 16 & 9 & 2 & 155 \\
\hline Brasil & 93 & 204 & 113 & 176 & 60 & 194 & 50 & 57 & 947 \\
\hline Chile & 24 & 17 & 19 & 4 & & 1 & 4 & 1 & 70 \\
\hline Colombia & 12 & 3 & 6 & 4 & 2 & 2 & 3 & & 32 \\
\hline Costa Rica & 25 & 2 & 7 & 2 & 3 & 4 & & 1 & 44 \\
\hline Cuba & 15 & & 30 & & & & 2 & & 47 \\
\hline México & 84 & 88 & 130 & 75 & 7 & 50 & 26 & 27 & 487 \\
\hline Perú & 7 & & 1 & 2 & & 1 & 1 & & 12 \\
\hline Venezuela & 4 & 67 & 127 & 16 & 19 & 21 & 27 & & 281 \\
\hline Total & 309 & 417 & 459 & 295 & 96 & 289 & 122 & 88 & 2.075 \\
\hline
\end{tabular}

Fuente: Oficina de Patentes de los Estados Unidos (USPTO). Elaboración de los autores.

En la Tabla 7 se observa que el área donde más contribuyen las mujeres de los países en estudio es en la de Química y Metalurgia con 18,34\%. Venezuela y Cuba presentan valores mayores al promedio de los otros países con respecto a esa sección, mientras que Chile, después de Perú, es el país que está más lejos del promedio en esa área. 
La tabla 7 también muestra que en Brasil, Chile y Cuba el género femenino contribuye más en el área de Necesidades Humanas que en otras áreas en la producción de patentes. Para el resto de los países no existe dicha coincidencia, se observa que en Argentina las mujeres contribuyen más en la producción de patentes asociadas al área Química y Metalurgia mientras que en Colombia lo hacen hacía el área de Ingeniería Mecánica. En Costa Rica el género femenino aporta más al área de Construcciones Fijas, mientras que las inventoras mexicanas lo hacen hacía el área de Física y las venezolanas contribuyen más hacia el área de Operaciones y Transporte.

TABLA 7

Contribución de las mujeres en las patentes otorgadas por secciones CIP Países seleccionados - 1990-2006

\begin{tabular}{|c|c|c|c|c|c|c|c|c|}
\hline \multicolumn{9}{|c|}{ En porcentaje } \\
\hline Países & $\begin{array}{c}\text { Química } \\
\text { y Meta- } \\
\text { lurgia }\end{array}$ & $\begin{array}{c}\text { Necesi- } \\
\text { dades } \\
\text { Humanas }\end{array}$ & $\begin{array}{l}\text { Tex- } \\
\text { tiles }\end{array}$ & Física & $\begin{array}{c}\text { Opera- } \\
\text { ciones y } \\
\text { Transporte }\end{array}$ & $\begin{array}{c}\text { Const. } \\
\text { Fijas }\end{array}$ & $\begin{array}{c}\text { Inge- } \\
\text { niería } \\
\text { Mecánica }\end{array}$ & $\begin{array}{l}\text { Electri- } \\
\text { cidad }\end{array}$ \\
\hline Argentina & 18,33 & 5,40 & 6,25 & 2,22 & 0,00 & 0,00 & 0,00 & 0,00 \\
\hline Brasil & 14,85 & 18,26 & 18,79 & 10,13 & 4,79 & 3,05 & 3,19 & 3,12 \\
\hline Chile & 7,40 & 22,15 & 0,00 & 16,66 & 0,00 & 0,00 & 1,00 & 0,00 \\
\hline Colombia & 10,18 & 1,66 & 0,00 & 11,11 & 0,00 & 0,00 & 50,00 & 0,00 \\
\hline Costa Rica & 16,93 & 0,00 & 0,00 & 0,00 & 0,00 & 66,66 & 0,00 & 0,00 \\
\hline Cuba & 39,86 & 42,95 & 0,00 & 7,14 & 0,00 & 0,00 & 0,00 & 0,00 \\
\hline México & 10,33 & 7,17 & 1,44 & 14,05 & 3,37 & 0,00 & 0,00 & 1,38 \\
\hline Perú & 0,00 & 0,00 & 0,00 & 0,00 & 0,00 & 0,00 & 0,00 & 0,00 \\
\hline Venezuela & 27,11 & 12,50 & 0,00 & 12,17 & 28,23 & 5,00 & 9,12 & 0,00 \\
\hline Promedio $\mathrm{d}$ & & & & & & & & \\
\hline la muestra & 18,34 & 12,15 & 12,14 & 10,86 & 7,69 & 4,98 & 3,64 & 2,52 \\
\hline
\end{tabular}

Fuente: Oficina de Patentes de los Estados Unidos (USPTO). Elaboración de los autores.

La Tabla 8 presenta el promedio de citas por participación de género del total de la muestra. Se calculó tomando el total de citas de las patentes registradas por participación de género y se dividió entre el número total de patentes por cada categoría. Se observa que las patentes producidas solo por mujeres o solo por hombres tienen un impacto promedio casi igual. Es decir, la calidad de una patente producida con la participación de mujeres solamente es casi la misma que aquellas donde participan los hombres solamente. 
Rosa María Morales Valera, Domingo Alberto Sifontes Fernandez

TABLA 8

Promedio de citas por patentes, según el género del inventor que patenta Países seleccionados (1) - 1990-2006

\begin{tabular}{l|c|c|c|c}
\hline $\begin{array}{c}\text { Género } \\
\text { del inventor }\end{array}$ & $\begin{array}{c}\text { Promedio } \\
\text { citas }\end{array}$ & $\begin{array}{c}\text { Desviación } \\
\text { estándar }\end{array}$ & Mínimo & Máximo \\
\hline Solo por mujeres & 3,32 & 4,08 & 0 & 18 \\
Solo por hombres & 3,7 & 6,21 & 0 & 87 \\
Ambos géneros & 2,88 & 4,67 & 0 & 27 \\
\hline
\end{tabular}

Fuente: Oficina de Patentes de los Estados Unidos (USPTO). Elaboración de los autores.

(1) Argentina, Brasil, Chile, Colombia, Costa Rica, Cuba, México, Perú, Venezuela.

La Tabla 9 muestra el impacto promedio de las patentes por participación de género en cada país estudiado. Se desprende que el impacto promedio de las patentes producidas solo por mujeres en la mayoría de los países de la muestra está por encima de 3 citas por patente, México y Colombia son la excepción. Para Argentina, Chile y Costa Rica se observa que el impacto promedio de las patentes producidas solo por mujeres es superior al producido solo por los hombres. Por el contrario, en Brasil, Colombia y Venezuela el promedio de las citas por patentes con participación solo del género femenino es menor a aquellas en las que solo participan hombres.

Con respecto a la calidad de las patentes, la tabla también muestra que de las registradas solo por mujeres Argentina posee la patente más citada con 18 citas. Venezuela posee las patentes más citadas en el caso de solo hombres y de colaboración mixta con 87 y 27 citas respectivamente.

Si se compara el promedio de citas por país (Tabla 9) con el promedio de citas por género de toda la muestra (Tabla 8), se observa que Venezuela es el único país que está por encima del promedio de citas de patentes registradas por ambos géneros. En el caso de las citas de las patentes otorgadas a inventores en la categoría de solo hombres, se observa que Argentina, México y Venezuela son los países cuyo promedio está por encima del exhibido por la muestra $(3,7$ citas por patente). 
TABLA 9

Impacto promedio de las patentes, según género Países seleccionados - 1990-2006

\begin{tabular}{|c|c|c|c|c|c|c|}
\hline Países & Géneros & \begin{tabular}{|c|} 
Número \\
total de \\
patentes
\end{tabular} & \begin{tabular}{|c|} 
Promedio \\
de citas por \\
patente
\end{tabular} & $\begin{array}{l}\text { Desviación } \\
\text { estándar }\end{array}$ & $\begin{array}{l}\text { Míni- } \\
\text { mo }\end{array}$ & $\begin{array}{c}\text { Máxi- } \\
\text { mo }\end{array}$ \\
\hline \multirow{3}{*}{ Argentina } & Solo mujeres & 4 & 5,75 & 8,18 & 1 & 18 \\
\hline & Ambos géneros & 12 & 0,58 & 0,99 & 0 & 3 \\
\hline & Solo hombres & 136 & 3,78 & 7,34 & 0 & 59 \\
\hline \multirow{3}{*}{ Brasil } & Solo mujeres & 33 & 3,09 & 3,81 & 1 & 13 \\
\hline & Ambos géneros & 127 & 2,55 & 5,49 & 0 & 24 \\
\hline & Solo hombres & 789 & 3,63 & 5,76 & 0 & 50 \\
\hline \multirow{3}{*}{ Chile } & Solo mujeres & 3 & 6,66 & 3,78 & 4 & 11 \\
\hline & Ambos géneros & 13 & 1,46 & 2,14 & 0 & 6 \\
\hline & Solo hombres & 51 & 2,86 & 5,4 & 0 & 35 \\
\hline \multirow{3}{*}{ Colombia } & Solo mujeres & 1 & - & - & - & - \\
\hline & Ambos géneros & 5 & 7 & 7,58 & 0 & 17 \\
\hline & Solo hombres & 26 & 2,26 & 4,79 & 0 & 24 \\
\hline \multirow{3}{*}{ Costa Rica } & Solo mujeres & 2 & 3,5 & 3,53 & 1 & 6 \\
\hline & Ambos géneros & 5 & 1,6 & 0,89 & 0 & 2 \\
\hline & Solo hombres & 37 & 1,21 & 3,48 & 0 & 15 \\
\hline \multirow{3}{*}{ Cuba } & Solo mujeres & - & - & - & - & - \\
\hline & Ambos géneros & 39 & 2,51 & 3,83 & 0 & 14 \\
\hline & Solo hombres & 8 & 3 & 7,28 & 0 & 21 \\
\hline \multirow{3}{*}{ México } & Solo mujeres & 12 & 2,83 & 4,8 & 0 & 17 \\
\hline & Ambos géneros & 39 & 2,43 & 3,38 & 0 & 10 \\
\hline & Solo hombres & 411 & 3,82 & 5,35 & 0 & 50 \\
\hline \multirow{3}{*}{ Perú } & Solo mujeres & - & - & - & - & - \\
\hline & Ambos géneros & - & - & - & - & - \\
\hline & Solo hombres & 12 & 3,08 & 4,03 & 0 & 14 \\
\hline \multirow{3}{*}{ Venezuela } & Solo mujeres & 19 & 3,15 & 3,16 & 0 & 12 \\
\hline & Ambos géneros & 105 & 3,87 & 4,99 & 0 & 27 \\
\hline & Solo hombres & 150 & 4,88 & 9,61 & 0 & 87 \\
\hline
\end{tabular}

Fuente: Oficina de Patentes de los Estados Unidos (USPTO). Elaboración de los autores. 


\section{Discusión y conclusiones}

La producción de inventos se encuentra concentrada en tres países: Brasil, México y Venezuela, con el $82,62 \%$ de las mismas y estos países presentan diferencias importantes entre sí: Brasil produce 2,06 veces más patentes que México y 3,47 veces más que Venezuela. Por su parte, México produce 1,69 veces más patentes que Venezuela. El 17,38\% restante se lo dividen 6 países: Argentina, Chile, Colombia, Costa Rica, Cuba y Perú.

Los resultados muestran una diferencia en la producción de patentes a favor de los hombres, independientemente que se cuenten las patentes de participación mixta, esto quiere decir que el género masculino domina la escena en la producción de patentes en América latina, al menos para el período de estudio analizado en la presente investigación. Esto coincide con Mauleón y Bordons (2010) para España y McMillan (2009) para Estados Unidos de Norteamérica. También se muestra una disminución de las patentes registradas solo por hombres y solo por mujeres a favor de la participación mixta. En 1990 las mujeres solas registraban el 4,92\% de las patentes y los hombres el 88,52\%, mientras que en el año 2006 pasaron a 2,78\% y $75 \%$ respectivamente, pasando las de participación mixta del 6,56\% en 1990 a $24,22 \%$ en 2006. Profundizar en las razones del incremento de la participación mixta sería un tema abordar en futuras investigaciones. Las mujeres (solo mujeres y mixtas) participaron en el 11,48\% de las invenciones en 1990 y en el $25 \%$ en 2006, mientras que los hombres (solo hombres y mixtas) lo hicieron en el 95,08\% en 1990 y en el $97,22 \%$ en 2006 , representando un crecimiento mayor en la participación de las mujeres que en la de hombres. A pesar de que la participación femenina ha aumentado en el registro de las invenciones, aún la brecha entre hombres y mujeres es alta. Ahora bien, ¿Es importante disminuir esa brecha en América Latina? ¿Una menor desigualdad garantiza un aumento en la productividad de las organizaciones y de los países? Estudios en empresas Europeas (TURNER, 2006) muestran que una mayor diversidad de género, no solo impulsa la productividad del género femenino sino también del equipo de trabajo o de las organizaciones con mayor diversidad, de allí, la importancia de disminuir la desigualdad. Asimismo, en este estudio se observa que la calidad de las invenciones es bastante similar para ambos géneros, no hay mayor diferencia entre aquellas donde hay participación solo del género femenino o solo del género masculino. Por lo tanto, una disminución de la desigualdad de género podría impactar positivamente el desempeño innovador de las organizaciones de distintas formas. 
La realidad Latinoamericana muestra semejanzas respecto a Europa en lo referido a la contribución de las mujeres en la actividad patentadora, es decir, las mujeres patentan menos que los hombres, sin embargo, la participación femenina ha venido en aumento en los últimos años pero no ha sido completamente utilizada, tal como señala Busolt y Kugele (2009). Por otra parte, la contribución femenina de acuerdo al país de origen en América latina es similar a la Europea, exceptuando Cuba y Venezuela, que presentan en términos promedio una contribución mayor a la de Latinoamericana y Europa.

La explicación de la diferencia en la contribución femenina en la producción de patentes para Europa y otros países desarrollados se ha centrado en el costo del cuidado diario de los niños Chiuri (2000) y en la diferencia de ingresos entre países Frietsch et al. (2009). Estos argumentos son poco probables de aplicar en América Latina. Los costos de cuidado diario de los niños para todos los países son bastantes bajos y homogéneos, hay que recordar que culturalmente este tipo de trabajo es remunerado a nivel de salario mínimo y es bastante probable que las inventoras puedan pagarlo. Por su parte, la diferencia de ingresos se descarta como una causa de la diferencias en la contribución femenina por países. Datos del Banco Mundial revelan que para el período de estudio, Cuba y Venezuela representan el séptimo y el quinto lugar en cuanto a ingreso per-cápita se refiere dentro de los países de la muestra y a su vez presentan la mayor contribución femenina. Por otra parte, Argentina y México, ocupan el primer y segundo lugar en términos de ingresos (medido a través del PIB per capita), mientras que su posición en cuanto a contribución femenina, son la séptima y la octava, respectivamente. El único país que mantiene una relación consistente entre ingresos y contribución femenina es Perú, ya que en ambos casos, ocupa el último lugar. La discusión anterior refleja la necesidad de investigación para explicar las causas de las diferencias en la contribución femenina en producción de patentes en Latinoamérica.

Algunos de los argumentos usados en la literatura para explicar las razones de la escasa contribución de las mujeres en el registro de patentes son las dificultades en el acceso a entrenamiento técnico y matemático así como la evidencia de que menos mujeres eligen carreras y participan en el área de ciencias físicas e ingeniería (DURACK, 1997; BURK, 2011). Estos argumentos podrían usarse para el caso de América Latina. Una de las posibles razones por las cuales se presentan diferencias en la contribución femenina entre los países de América Latina podría ser las desigualdades en el acceso al entrenamiento técnico necesario que podría originar una menor participación femenina en el personal de ciencia 
y tecnología. Asi, Cuba tiene una mayor contribución del género femenino en las invenciones (39,45\%), y de acuerdo a estadísticas del RICYT, entre el 50 y 53\% del personal de ciencia y tecnología pertenece al género femenino. Por otro lado, en Chile la contribución femenina en el registro de patentes alcanza un $12 \%$ y las mujeres representan un 30\% del personal de ciencia y tecnología. Un mayor acceso a entrenamiento técnico y a posiciones en el sector de la ciencia y tecnología, podría disminuir la brecha en la desigualdad de contribución femenina en el registro de invenciones en América Latina.

El área de Química y Metalurgia es la de mayor contribución femenina. Esto coincide con Giuri et al. (2007), Naldi et al. (2004) y Whittington y Smith-Doerr (2008). En sectores asociados al sector químico, las mujeres tienden a contribuir más. Por otra parte, el área en la que menos contribuyen las inventoras es en la de Electricidad. Por género, se tiene que Brasil es el país que más patentes posee en cada categoría, presentando diferencias importantes con cada uno de los países que forman parte del estudio. Esta diferencia varía para cada país y depende de muchos factores. Es importante destacar que existen diferencias entre países en cuanto a la contribución femenina por área. No todos los países coinciden en las áreas de especialización de las mujeres. Así se observa que Brasil, Chile y Cuba coinciden en que las mujeres contribuyen más en el área de Necesidades Humanas. Este resultado es similar al de Mauleón y Bordons (2010) para el caso de España. El resto de los países de la muestra no tiene el mismo comportamiento. Frietsch et al. (2009) han encontrado que la brecha entre países con respecto a las áreas de especialización de las mujeres puede estar asociada a las diferencias inter industriales de los países ó al tipo de área en la que las mujeres suelen comprometerse más. Es posible que en América Latina la distancia entre las especializaciones por país también se deba a las áreas en las que las mujeres han tenido más incentivos para hacer investigación, como también, al acceso en igualdad de condiciones de determinadas industrias.

Finalmente, los resultados muestran que por el tipo de organización, las privadas registran más patentes que las públicas y las mixtas, así mismo la participación es principalmente de solo hombres en los tres tipos de organización. Tanto los hombres como las mujeres patentan más en el sector privado, sin embargo, la desigualdad de género en este sector existe y es mayor respecto al sector público. En efecto, se tiene que en las organizaciones privadas los hombres patentan hasta 27 veces más que las mujeres, en las públicas, lo hacen por casi 9 veces más que las mujeres y en las mixtas la diferencia llega a hacer de 38, corroborando lo planteado por Whittington y Smith-Doerr (2008) respecto a la poca flexibilidad de las organizaciones 
privadas no relacionadas a las ciencias de la vida y su relación con la participación de las mujeres en la actividad patentadora. Por otra parte, se observa que cuando en el invento participan personas de un solo género independientemente de cuál sea, existe mayor probabilidad que la patente sea propiedad de un ente privado, mientras que cuando la participación es mixta, la probabilidad es mayor para los entes públicos, esto permite pensar que existe un mayor incentivo dentro de las organizaciones públicas para patentar cuando la participación es mixta y también en la existencia de mayores presencia y facilidades para la incorporación de las mujeres.

Las dificultades metodológicas del estudio se centran en los problemas para deducir el género de los autores y el tipo de esquema de propiedad de la entidad que patenta. El primer problema está relacionado con el hecho de que algunas veces el nombre de los autores no se presenta en español ó que del primer nombre no se podía deducir el género. La segunda dificultad está asociada a que en algunos casos se hizo complicado descifrar si la entidad dueña de la patente era pública o privada, esto por el escaso conocimiento de empresas de otros países ó por la imposibilidad de no encontrar un registro de la empresa vía internet. Próximas investigaciones pueden dirigir sus esfuerzos a estudiar las causas de las diferencias de género en la actividad patentadora en cada uno de los países y las diferencias existentes en América latina entre el total de inventores y el total de investigadores, entendiendo que no todos los investigadores generan patentes. Partiendo de este hecho, sería importante estudiar las diferencias de género entre un grupo y otro para analizar en cual las mujeres tienen mayor participación y ¿por qué?

\section{Referencias}

BUSOLT, U.; KUGELE, K. The gender innovation and research productivity gap in Europe. International Journal Innovation and Sustainable Development, v. 4, n. 2/3, p. 109-122, 2009.

BURK, D. Do patents have gender? Journal of Gender, Social Policy \& the Law, v. 19, n. 3 , p. 881-919, 2011.

CHIURI, M. Quality and demand of child care and female labour supply in Italy. Labour, v. 14 , n. 1 , p. $97-118,2000$.

DING, W. W.; MURRAY, F.; STUART, T. E. Gender differences in patenting in the academic life sciences. Science, v. 313, n. 5.787, p. 665-667, 2006.

DURACK, K. Gender, technology and the history of technical communication. Technical Communication Quarterly, v. 6, n. 3, p. 249-260, 1997. 
Rosa María Morales Valera, Domingo Alberto Sifontes Fernandez

ESTÉBANEZ, M. Un enfoque de género en la construcción de indicadores de ciencia y tecnología en la región interamericana/iberoamericana. El Estado de la Ciencia 2002. Ricyt, 2002.

FRIETSCH, R.; HALLER, I.; FUNKEN-VROHLINGS, M.; GRUPP, H. Gender-specific patterns in patenting and publishing. Research Policy, v. 38, n. 4, p. 590-599, 2009.

GIURI, P.; MARIANI, M.; BRUSONI, S.; CRESPI, G.; FRANCOZ, D.; GAMBARDELLA, A.; GARCIA-FONTES, W. et al. Inventors and invention processes in Europe: results from the PatVal-EU survey. Research Policy, v. 36, n. 8, p. 1107-1127, 2007.

HALBERT, D. Feminist interpretations of intellectual property. Journal of Gender, Social Policy \& the Law, v. 14, n. 3, p. 431-460, 2006.

KUGELE, K Patents invented by women and their participation in research and devolopment: a European comparative approach. En: GODFROY-GENIN, A.-S. (Ed.). Women in engineering and technology research. Proceedings of the PROMETEA Conference, París Octubre 26-27, 2007.

LÁSCARIS, T. Hacia la incorporación del enfoque de género en los indicadores de Ciencia y Tecnología en América Central. OEA-Ricyt, Agosto 2004.

MANI, S. Government, innovation and technology policy: an analysis of the Brazilian experience during the 1990s. United Nations University INTECH, 2001 (Discussion paper).

MAULEÓN, E.; BORDONS, M. Male and female involvement in patenting activity in Spain. Scientometrics, v. 83, n. 3, p. 605-621, 2010.

MCMILLAN, G. S. Gender differences in patenting activity: an examination of the US biotechnology industry. Scientometrics, v. 80, n. 3, p. 683-691, 2009.

NALDI, F.; LUZI, D.; VALENTE, A.; VANNINI, I. Scientific and technological performance by gender. En: MOED, H. F.; GLANZEL, W.; SCHMOCH, U. (Eds.). Handbook of quantitative science and technology research Kluwer Academic Publishers. 2004, p.299-314.

TURNER, L. Gender diversity and performance. Women in science and technology - The business perspective. Comunidad Económica Europea, 2006, p. 69-74.

VESSURI, H.; CANINO, M.; RAUSELL, M. Desarrollos metodológicos para la inclusión de la variable de género en la construcción de indicadores de ciencia, tecnología e innovación en la región iberoamericana. OEA-Ricyt, Septiembre 2004.

VESSURI, H.; CANINO, M. Igualdad entre géneros e indicadores de ciencia en Iberoamérica. El estado de la Ciencia 2006. Ricyt, 2006. 
WHITTINGTON, K. B.; SMITH-DOERR, L. Gender and commercial science: Women's patenting in the life sciences. The Journal of Technology Transfer, v. 30, n. 4, p. 355-370, 2005.

. Women inventors in context: disparities in patenting across academia and industry. Gender \& Society. 2008.

ENDEREÇOS PARA CORRESPONDENCIA

Rosa María Morales Valera - moralesr@uc.edu.ve

INFACES, Universidad de Carabobo

Av. Salvador Allende, Edif. Anexo Faces, PB

Naguanagua, Carabobo-2005

Venezuela

Domingo Alberto Sifontes Fernandez - dsifontes@uc.edu.ve

Escuela de Economía, Universidad de Carabobo

Av. Salvador Allende, Edif. Faces, 1er Piso.

Naguanagua, Carabobo-2005

Venezuela 
